\title{
Implicación de los gobiernos locales en la promoción del emprendimiento: evidencia para España
}

\author{
Luisa Cagica Carvalho*, Esteban Pérez Calderón**, María Pache Durán*** \\ *Universidad Aberta de Lisboa \\ Correo-e: luisam.carvalho@uab.pt | ORCID iD: https://orcid.org/0000-0002-9804-7813 \\ **Facultad de Ciencias Económicas y Empresariales, Universidad de Extremadura \\ Correo-e: estperez@unex.es | ORCID iD: https://orcid.org/0000-0002-4193-3769 \\ ***Facultad de Empresa, Finanzas y Turismo, Universidad de Extremadura \\ Correo-e: mpache@unex.es | ORCID iD: https://orcid.org/0000-0002-6670-5818
}

Recibido: 25-01-2018; 2a versión: 24-05-2018; Aceptado: 28-05-2018

Cómo citar este artículo/Citation: Cagica Carvalho, L.; Pérez Calderón, E.; Pache Durán, M. (2019). Implicación de los gobiernos locales en la promoción del emprendimiento: evidencia para España. Revista Española de Documentación Científica, 42 (1): e226. https://doi.org/10.3989/redc.2019.1.1559

Resumen: En el contexto de la divulgación de información de las administraciones locales, el presente estudio analiza, en primer lugar, el grado y el tipo de información sobre emprendimiento que los gobiernos locales de la provincia de Cáceres ofrecen a través de sus sitios web y, en segundo lugar, las posibles similitudes o diferencias existentes entre los municipios con respecto a sus niveles de divulgación. Los resultados muestran que el grado de información divulgado es bajo y que la información que más se divulga es la de carácter general, seguida de la información sobre emprendimiento digital y divulgación y comunicación con el emprendedor. La información divulgada sobre recursos y apoyo al emprendedor y emprendimiento activo, aunque existe, es muy escasa. El estudio también muestra la existencia de dos categorías de ayuntamientos en función del nivel de divulgación de información y sus características poblacionales, socio-económicas, fiscales y políticas que éstos presentan en cada una de ellas.

Palabras clave: divulgación de información; emprendimiento; gobiernos locales; indicadores; sitios web.

\section{Implication of local governments in the entrepreneurship promotion: evidence from Spain}

Abstract: This study analyzes, In the context of the dissemination of information from local administrations, the degree and type of information about entrepreneurship that local governments from the Cáceres province offer through their web pages and, secondly, the possible similarities and differences between municipalities regarding their disclosure levels. The results show that the smount of disclosed information is low and that the most widely disseminated information is general information, followed by information on digital entrepreneurship and dissemination and communication with the entrepreneur. Information on resources and support for the entrepreneur and active entrepreneurship is also disclosed, but is still very scarce. The study also reveals the existence of two categories of municipalities based on the level of disclosure of information, along with the population, socio-economic, fiscal and political characteristics for each of them.

Keywords: information disclosure; entrepreneurship; local governments; indicators; websites.

Copyright: $\odot 2019$ CSIC. Este es un artículo de acceso abierto distribuido bajo los términos de la licencia de uso y distribución Creative Commons Reconocimiento 4.0 Internacional (CC BY 4.0). 


\section{INTRODUCCIÓN}

El emprendimiento juega un papel esencial para la innovación, la creación de empleo, el crecimiento económico y el desarrollo local (Sullivan y Meek, 2012). La promoción del espíritu empresarial depende del acceso a la información y de políticas adecuadas dirigidas a los emprendedores (Sullivan y Meek, 2012). En países de gran dimensión, como es el caso de España, existen diferencias entre las provincias, en términos sociales, económicos y culturales $y$, aunque pueda haber una estrategia nacional de promoción del emprendimiento, se justifica una especial atención a nivel local en el diseño de políticas y ambientes contextuales que favorezcan la creación y el crecimiento de nuevas empresas. Uno de los problemas que enfrentan los emprendedores es el acceso a la información sobre las reglas, condiciones y oportunidades disponibles en una región para el desarrollo de su empresa.

Por otro lado, también se sabe que las Tecnologías de la Información y la Comunicación (TIC) están cada vez más difundidas y son utilizadas para la recogida de información por (1) ser de fácil acceso, (2) permitir una consulta previa y la preparación de la información, y (3) por ser un medio popularmente usado por la llamada generación de los Millenium (esta generación, de 18 a 34 años, se sitúa en un rango de edad en el que muchos jóvenes entran en el mercado de trabajo y a veces intentan crear su empresa) (Bonsón y Escobar, 2004). En este contexto, Baptista (2005) señala que el uso de las TIC está siendo hoy en día uno de los objetivos principales de los gobiernos europeos para ofrecer servicios públicos a través de Internet y otros canales electrónicos. Las administraciones públicas deben esforzarse para innovar, proporcionando y divulgando información relevante a los emprendedores de sus regiones y a potenciales emprendedores que busquen un lugar adecuado para su inversión, a través del uso de las TIC (Bekkers y otros, 2006). Los sitios web son actualmente la forma más popular en el ámbito de las TIC utilizados por las administraciones para la divulgación de información (Moneva y Martín, 2012; Nevado y otros, 2013).

La divulgación de información a través de sitios web está siendo adoptada cada vez más por las organizaciones (Joseph y Taplin, 2011; Moneva y Martín, 2012; Nevado y otros, 2013). Esta práctica permite una mayor proximidad entre las instituciones y los ciudadanos y una mejor comprensión de sus necesidades (Rodríguez y otros, 2010). Existen numerosos estudios que analizan la información divulgada por las administraciones a través de sus sitios web. La mayoría de las investigaciones se centran en la divulgación de información voluntaria ${ }^{1}$, financiera, sobre Sostenibilidad o Responsabilidad Social (RS). Sin embargo, los estudios sobre la difusión de información sobre emprendimiento, son todavía escasos.

La unidad de análisis en el estudio han sido los gobiernos locales. Esta elección se debe a la posición ventajosa que ocupan a la hora de conocer las demandas informativas de los ciudadanos (Díaz, 2009) gracias a su cercanía (Navarro y otros, 2010; Cueto y otros, 2014). Concretamente, se han elegido los 213 gobiernos locales que conforman la región de Cáceres, pertenecientes a la Comunidad Autónoma de Extremadura. La elección de esta Comunidad Autónoma obedece a la elevada tasa de paro que ha registrado en 2017, la más alta del país, con un $28,31 \%$, según los datos de la Encuesta de Población Activa (INE, 2017). Teniendo esto en cuenta, es relevante estudiar hasta qué punto los municipios pueden contribuir a revertir esta tendencia, a través de la promoción del emprendimiento y los ecosistemas empresariales.

Este trabajo tiene como objetivo, por un lado, el análisis de la información sobre emprendimiento que los municipios ofrecen a sus ciudadanos, es decir, se plantea analizar el grado y el tipo de divulgación de la información que los gobiernos locales vienen realizando a los emprendedores. A partir de una relación de indicadores construida tomando como referencia la literatura existente, se realiza un análisis exhaustivo de los sitios web de los municipios, con el propósito de detectar la presencia o ausencia de la información de cada indicador, mediante la utilización de la técnica de análisis de contenido. Posteriormente, se utilizan diferentes técnicas estadísticas para identificar las posibles similitudes o diferencias existentes entre los gobiernos locales de la muestra con respecto a sus niveles de divulgación.

Los resultados muestran que el grado de divulgación de información sobre emprendimiento de los municipios cacereños es bajo, siendo la información de carácter general y la relativa a emprendimiento digital y comunicación con el emprendedor, las más divulgadas. La información sobre recursos y apoyo al emprendedor y emprendimiento activo, aunque existe, es muy escasa. No obstante, a pesar de que estos resultados reflejan una escasa divulgación, ponen de manifiesto la existencia de una iniciativa de difusión por parte de los gobiernos locales cacereños de este tipo de información, tan importante para el crecimiento económico. El estudio también muestra la existencia de dos categorías de ayuntamientos en función del nivel de divulgación de información y las características poblacionales, socio-económicas, fiscales y políticas que éstos presentan en cada una de ellas. 
El trabajo se estructura como sigue. Tras esta introducción, se lleva a cabo una revisión de la literatura sobre la divulgación de información a través de los sitios web. Seguidamente, se indica la metodología empleada y, finalmente, se comentan los resultados y las conclusiones del estudio.

\section{REVISIÓN DE LA LITERATURA}

\section{Innovación en el sector público}

La innovación en sentido general puede ser entendida como un nuevo o mejorado producto, servicio, proceso de producción, de distribución, o incluso un nuevo modelo de negocio. Cuando nos referimos al sector público, puede ser considerada como un proceso en curso (Batalli, 2011). La innovación entendida como un proceso en curso puede referirse, por ejemplo, a la descentralización de la Administración Pública, a la simplificación de los procedimientos, a la informatización de los servicios y a las mejoras en la organización de los recursos humanos. Así podemos considerar que la innovación en el proceso en la Administración Pública engloba la creación de mecanismos que aseguren la transparencia del sector público, en particular en términos de contabilidad, presupuesto, compras públicas e incluso en términos de atracción de la inversión privada. Al ser considerada como un proceso, la innovación en este sector nunca está cerrada o completa, es siempre una solución abierta que puede ser transformada y mejorada por sus adoptivos.

La introducción de e-services en la Administración Pública en los últimos años constituye una de sus principales innovaciones, pues permite poner a disposición de sus ciudadanos de manera más eficiente y efectiva un conjunto de servicios (OECD, 1999). La disponibilidad de e-services implica no sólo cambios en procesos tecnológicos, sino también cambios en su estructura organizacional. La digitalización de la información administrativa es una herramienta que puede hacer que el trabajo de las administraciones públicas sea más visible para los ciudadanos (Dunleavy y otros, 2006). La Administración Pública está desarrollando en todo el mundo sistemas de información y servicios electrónicos que satisfagan las necesidades y la demanda de los ciudadanos.

En el caso de los municipios, objeto de esta investigación, la aplicación de las TIC presenta tres dimensiones (OSI y LGPSRI, 2007):

- Government to Government (G2G) - se refiere al uso de las TIC para mejorar el funcionamiento de la Administración Pública.
- Governement to Citizens (G2C) - se refiere a la prestación de servicios a los ciudadanos.

- Government to Business (G2B) - se refiere a la oferta de servicios para las empresas.

Este estudio se sitúa en la última dimensión, es decir, en la referida a los servicios y a la información ofrecida a las empresas, en particular a los emprendedores, para facilitar su proceso de toma de decisiones, concretamente en la fase de creación del negocio.

\section{Emprendedor e información asimétrica}

El emprendedor está permanentemente ante la necesidad de tomar decisiones, pero no siempre dispone de toda la información que le permita adoptar la mejor elección a largo plazo. El problema de la información asimétrica es un fallo de mercado que debe ser minimizado por el estado. El acceso a la información es a menudo imperfecto e implica costes para su obtención (Stiglitz, 2000). En el marco de los estudios sobre emprendimiento, la información imperfecta ha venido a prestar especial atención a la elección del emprendedor entre crear y no crear una empresa (Lee y Venkataraman, 2006). La literatura muestra dos mecanismos para disminuir la asimetría de la información: signaling (señalización) y screening (estrategia para combatir la selección adversa) (Stiglitz, 2000). El screening se refiere a las iniciativas llevadas a cabo por el agente desinformado (la víctima de la información asimétrica) para acceder a la información y al conocimiento, mientras que, en el signaling, los agentes informados, son quienes hacen el primer movimiento para diferenciarse.

En este contexto, el emprendedor tiene que tener en cuenta la información puesta a su disposición en la toma de decisiones y, al parecer, esa disponibilidad de información, en términos regionales, está asociada a la tasa de emprendimiento regional (Minniti, 2004). Teniendo en cuenta que parte de esta información regional a disposición de los emprendedores es divulgada a través de los municipios, es esencial estudiar el tipo y el grado de información que ofrecen los gobiernos locales a los emprendedores a través de los sitios web.

\section{Divulgación de información a través de los sitios web}

Existen múltiples estudios sobre divulgación de información en el entorno empresarial, así como de los principales factores explicativos de la política de divulgación (Navarro y otros, 2010). La divulgación de información a través de sitios web está siendo adoptada cada vez más por las organizaciones y, 
en los últimos años, se han incrementado los estudios en el sector público (Lodhia y otros, 2012; Cueto y otros, 2014; Navarro y otros, 2016), concretamente en la Administración Local. Esta práctica proporciona una mayor y mejor comprensión de las necesidades de los ciudadanos, debido a la creciente demanda de información generada en los últimos años (Rodríguez y otros, 2010).

Muchos estudios han analizado el tipo y el grado de información divulgada por las administraciones a través de los sitios web. Algunas de estas investigaciones se han centrado en la divulgación de información voluntaria y financiera (Caba y otros, 2005; Laswad y otros, 2005, y Cárcaba y García, 2008). Otras, como las de Farneti y Guthrie (2009), Nevado y otros (2013) y Navarro y otros (2015), estudian la divulgación de información sobre Sostenibilidad o RS en entidades públicas. La Tabla I muestra un resumen de los trabajos más recientes.

Por otro lado, hay que mencionar en España, la existencia del Índice de Transparencia de los Ayuntamientos (ITA) elaborado por Transparency International España ${ }^{2}$, que constituye una herramienta para medir y evaluar comparativamente el nivel de información pública y accesible que ofrecen las entidades locales a los ciudadanos y la sociedad. Este índice trata de propiciar un mayor acercamiento de los ayuntamientos a los ciudadanos, fomentando el aumento de la información que éstos reciben de las entidades locales, tanto de la situación de los propios ayuntamientos, como sobre las actividades que realizan, y también sobre las prestaciones y servicios a los que pueden acceder los propios ciudadanos. No podemos olvidar que en el mundo de la Documentación como disciplina académica se ha trabajado mucho en el análisis de la información de los sitios web. Entre estos trabajos podemos citar los de Ferreira y de Barros, 2011; González y otros, 2012; Hilera y otros, 2013; Pereira y otros, 2013; Casasola y otros, 2017; Martínez y Berrocal, 2017; Piñeiro y otros, 2017; Gutiérrez-Ponce y otros, 2018.

Sin embargo, los estudios sobre la difusión de información relacionada con la capacidad empresarial de las administraciones públicas son todavía escasos (Carvalho y otros, 2017). De ahí la originalidad de este trabajo, que permitirá, por un lado, obtener evidencia empírica y contribuir a la

Tabla I. Estudios sobre divulgación de información

\begin{tabular}{|c|c|c|c|}
\hline $\begin{array}{c}\text { Tipo de } \\
\text { información } \\
\text { analizada }\end{array}$ & Ámbito & Estudios & Muestra analizada \\
\hline \multirow{8}{*}{$\begin{array}{l}\text { Financiera y } \\
\text { voluntaria }\end{array}$} & \multirow{4}{*}{ Internacional } & Rodríguez y otros (2006) & $\begin{array}{c}\text { Gobiernos anglosajones, de América del Sur } \\
\text { y Centro Europa }\end{array}$ \\
\hline & & Yu (2010) & Gobiernos locales de China \\
\hline & & Mendes y otros (2016) & Municipios portugueses \\
\hline & & $\begin{array}{l}\text { Oates y Moradi-Motlagh } \\
(2016)\end{array}$ & Gobiernos locales en Australia \\
\hline & \multirow{4}{*}{ Nacional } & Rodríguez y otros (2007) & Gobiernos españoles \\
\hline & & Caba y otros (2008) & Municipios españoles \\
\hline & & Serrano y otros (2009) & $\begin{array}{l}\text { Municipios y capitales de } \\
\text { más de } 75.000 \text { habitantes }\end{array}$ \\
\hline & & Cárcaba y García (2010) & Municipios de más de 20.000 habitantes \\
\hline \multirow{8}{*}{$\begin{array}{l}\text { Sostenibilidad } \\
\text { y RS }\end{array}$} & \multirow{4}{*}{ Internacional } & Mir y otros (2015) & Gobiernos locales de Nueva Zelanda \\
\hline & & Navarro y otros (2015) & Municipios de los Países Nórdicos \\
\hline & & Brusca y otros (2016) & Gobiernos locales italianos y 59 españoles \\
\hline & & Hashim y otros (2016) & Entidades locales en Malasia \\
\hline & \multirow{4}{*}{ Nacional } & $\begin{array}{l}\text { Prado-Lorenzo y otros } \\
(2012)\end{array}$ & $\begin{array}{l}\text { Municipios españoles de más de } 100.000 \\
\text { habitantes }\end{array}$ \\
\hline & & $\begin{array}{l}\text { García-Sánchez y otros } \\
(2013)\end{array}$ & Municipios españoles \\
\hline & & $\begin{array}{l}\text { Cuadrado-Ballesteros } \\
(2014)\end{array}$ & Municipios españoles \\
\hline & & $\begin{array}{l}\text { Alcaraz-Quiles y otros } \\
\text { (2015) }\end{array}$ & Gobiernos locales españoles \\
\hline
\end{tabular}


generación de conocimiento sobre divulgación de información por parte de los gobiernos. Y, por otro lado, poner de manifiesto la necesidad de promover una actitud positiva de los gobiernos locales hacia el espíritu empresarial y la oportunidad que brindan los sitios web para contribuir al desarrollo económico y al aumento del empleo.

\section{METODOLOGÍA}

Esta investigación se centra en el análisis del grado y del tipo de información sobre emprendimiento que divulgan los 213 gobiernos locales que conforman la provincia de Cáceres. Los principales argumentos que respaldan esta decisión son los siguientes: Es una región con gran potencial para proyectos empresariales debido a su buena posición geográfica y accesibilidad. En ella destacan como sectores estratégicos en los que potenciar el emprendimiento, la agricultura, la tecnología, la salud y la calidad de vida, la cultura y el turismo, los servicios, la industria y la energía. Sin embargo, existen desafíos importantes que la región aún enfrenta, como el hecho de que debe orientarse hacia un mayor crecimiento económico y del empleo, a la implementación de un modelo de turismo sostenible y al fortalecimiento de grandes infraestructuras, entre otros. Además, es una de las regiones menos desarrolladas, con bajas tasas de iniciativa empresarial y una elevada tasa de población envejecida. La tasa de paro registrada en 2017 es la más alta del país, con un 28,31\%, según los datos de la Encuesta de Población Activa (INE, 2017). Por otro lado, a pesar de formar parte de una Comunidad Autónoma reconocida como la Región Emprendedora Europea 2017 por construir un ecosistema de apoyo a este sector y a la empresa, Cáceres, aun siendo la segunda región más extensa de España, tan sólo cuenta con dos puntos de acompañamiento empresarial (PAE), uno en el municipio de Cáceres y otro en Plasencia, para ofrecer orientación en la construcción de proyectos empresariales (información sobre permisos y normativas, opciones de financiación, ayudas económicas públicas, asesoramiento para expansión a nuevos mercados e internacionalización, gestión de las actividades formativas). Por todo ello, resulta de gran relevancia estudiar hasta qué punto los municipios de la provincia extremeña de Cáceres están desarrollando una adecuada labor de difusión mediante el empleo de las TICs para contribuir a la promoción del emprendimiento y de los ecosistemas empresariales y, por tanto, al crecimiento económico de la región.

Para conseguir los objetivos del estudio, comenzamos recabando información mediante la utilización de los datos disponibles en los sitios web de los municipios durante los meses de septiembre y octubre de 2017. La información se obtuvo utilizando la técnica del análisis de contenido empleada en numerosos estudios de esta naturaleza (Moneva y Martín, 2012; Nevado y otros, 2013; Navarro y otros, 2015; Beuren y Angonese, 2015). Para recoger la información se ha accedido al buscador y al mapa web de página principal de cada municipio. En caso de no contener el mapa, se ha realizado la búsqueda a partir de los enlaces de la página principal. El instrumento utilizado para la recopilación de información ha sido el cuestionario propuesto por Carvalho y otros (2017), compuesto por un total de 49 indicadores divididos en 5 dimensiones (Tabla II). El sistema de puntuación utilizado consistió en asignar a cada indicador el valor 1 si la entidad revela la información relativa a él, y 0 en caso contrario (Gandía y Archidona, 2008; Jorge y otros, 2011; Frías-Aceituno y otros, 2013; Nevado y Gallardo, 2016).

Tabla II. Indicadores por dimensiones

\begin{tabular}{|l|l|c|}
\hline Dimensiones & \multicolumn{1}{|c|}{ Definición } & $\begin{array}{c}\mathbf{N}^{\circ} \text { de } \\
\text { indicadores }\end{array}$ \\
\hline Dimensión 1 & Información general & 10 \\
\hline Dimensión 2 & $\begin{array}{l}\text { Recursos y apoyo al } \\
\text { emprendedor }\end{array}$ & 12 \\
\hline Dimensión 3 & $\begin{array}{l}\text { Emprendimiento } \\
\text { activo }\end{array}$ & 10 \\
\hline Dimensión 4 & $\begin{array}{l}\text { Emprendimiento } \\
\text { digital }\end{array}$ & 6 \\
\hline Dimensión 5 & $\begin{array}{l}\text { Divulgación y } \\
\text { comunicación con el } \\
\text { emprendedor }\end{array}$ & $\mathbf{4 9}$ \\
\hline & Total de indicadores & \\
\hline
\end{tabular}

Fuente: Adaptado de Carvalho y otros (2017).

Además, de los índices correspondientes a las 5 dimensiones que se muestran en la Tabla II, se empleó un índice de divulgación total cuya consistencia interna se estimó con el coeficiente alfa de Cronbach (Cronbach, 1951). El valor de este coeficiente muestra (Tabla III y Tabla IV) la existencia de consistencia en las puntuaciones de los distintos índices que componen el índice total, ya que su valor, superior a 0,7, se considera aceptable (George y Mallery, 2003, p. 231). Estos índices individuales están altamente correlacionados entre sí.

Tabla III. Fiabilidad índice total

\begin{tabular}{|c|c|c|}
\hline $\begin{array}{c}\text { Alfa de } \\
\text { Cronbach }\end{array}$ & $\begin{array}{c}\text { Alfa de Cronbach } \\
\text { basada en los } \\
\text { elementos tipificados }\end{array}$ & $\begin{array}{c}\text { N. de } \\
\text { elementos }\end{array}$ \\
\hline 0,736 & 0,793 & 5 \\
\hline
\end{tabular}


Tabla IV. Estadísticos total-elemento

\begin{tabular}{|l|c|c|c|c|c|}
\hline & $\begin{array}{c}\text { Media de la } \\
\text { escala si se } \\
\text { elimina el } \\
\text { elemento }\end{array}$ & $\begin{array}{c}\text { Varianza de } \\
\text { la escala si } \\
\text { se elimina el } \\
\text { elemento }\end{array}$ & $\begin{array}{c}\text { Correlación } \\
\text { elemento- } \\
\text { total } \\
\text { corregida }\end{array}$ & $\begin{array}{c}\text { Alfa de } \\
\text { Correlación } \\
\text { múltiple al } \\
\text { cuadrado }\end{array}$ & $\begin{array}{c}\text { Cronbach si } \\
\text { se eleimina } \\
\text { el elemento }\end{array}$ \\
\hline $\begin{array}{l}\text { Indice de divulgación } \\
\text { de cada ayuntamiento } \\
\text { dimensión 1 }\end{array}$ & 7,5416 & 54,452 & 0,382 & 0,160 & 0,729 \\
\hline $\begin{array}{l}\text { Indice de divulgación } \\
\text { de cada ayuntamiento } \\
\text { dimensión 2 }\end{array}$ & 11,9845 & 47,992 & 0,665 & 0,699 & 0,643 \\
\hline $\begin{array}{l}\text { Indice de divulgación } \\
\text { de cada ayuntamiento } \\
\text { dimensión 3 }\end{array}$ & 12,5087 & 53,331 & 0,631 & 0,543 & 0,677 \\
\hline $\begin{array}{l}\text { Indice de divulgación } \\
\text { de cada ayuntamiento } \\
\text { dimensión 4 }\end{array}$ & 7,9156 & 36,714 & 0,425 & 0,193 & 0,790 \\
\hline $\begin{array}{l}\text { Indice de divulgación } \\
\text { de cada ayuntamiento } \\
\text { dimensión 5 }\end{array}$ & 11,5868 & 43,549 & 0,666 & 0,659 & 0,625 \\
\hline
\end{tabular}

En cuanto a la fiabilidad de la codificación, tal como justifica Abela (2000) en su trabajo sobre las técnicas de análisis de contenidos, los datos sobre fiabilidad exigen que dos codificadores, como mínimo, según indica Krippendorff (1990), describan de forma independiente un conjunto posiblemente amplio de unidades de registro en los términos de un lenguaje común. La fiabilidad se expresa como una función del acuerdo alcanzado entre los codificadores sobre la asignación de las unidades a las diversas categorías. Si dicho acuerdo es total para todas las unidades, está garantizada la fiabilidad, por el contrario, si no es mayor que el correspondiente al azar, la fiabilidad es nula. En este estudio, han sido dos los codificadores encargados de la recogida de datos, y el método empleado para el cálculo del acuerdo entre ambos ha sido el porcentaje de acuerdo. Ésta, es una de las mediciones más comúnmente reportadas por los estudios que realizan el cálculo de acuerdo (Peña, 2012), a pesar de las críticas recibidas por no dar cuenta del acuerdo entre codificadores alcanzado sólo por azar (Krippendorff, 2004; Lombard y otros, 2002). Los dos codificadores mostraron estar de acuerdo en más de un $87 \%$ de las codificaciones, realizadas en unas pruebas previas a la codificación individual.

Con la información recopilada por ambos codificadores y siguiendo la metodología utilizada en Carvalho y otros (2017), se elaboraron unos índices de divulgación con el objetivo de analizar el grado y el tipo de divulgación de información sobre emprendimiento que los municipios cacereños muestran en sus webs. El uso de índices para medir el nivel de información ha sido utilizado en los estudios de Gandía y Archidona (2008), Jorge y otros (2011),
Navarro y otros (2010 y 2015), Joseph y Taplin (2011), Moneva y Martín (2012) y Beuren y Angonese (2015), entre otros. Se parte de un índice que mide la divulgación por municipio en cada uno de los ejes de análisis (IDMD). A partir de este índice se calcula uno nuevo, que mide la información total en cada municipio (IDM). Por otro lado, se emplea un índice que mide la divulgación total de cada indicador (IDI), a partir del cual se construye otro para cada uno de las cinco dimensiones (IDD). Por último, el índice de divulgación total (IDT), que mide la divulgación total de la muestra (Tabla V).

Una vez calculados los índices, con el objeto de identificar posibles similitudes o diferencias existentes entre los gobiernos locales de nuestra muestra con respecto a sus niveles de divulgación, se ha realiza un Análisis de Conglomerados o Análisis Cluster. Este análisis es una herramienta exploratoria de análisis de datos que clasifica las observaciones en grupos relativamente homogéneos, llamados conglomerados (Jobson, 1992). El objetivo principal de esta metodología es agrupar aquellas observaciones que muestran el mayor grado de homogeneidad posible dentro del mismo grupo, mientras que al mismo tiempo representan grupos muy heterogéneos. Al hacer esto, aquellos individuos que sean similares pertenecerán al mismo grupo y aquellas observaciones que presenten características diferentes se agruparán en un grupo diferente. Según Jain (2010), la aplicación del análisis de conglomerados nos permitirá conocer más sobre los datos para poder establecer hipótesis, identificar en qué medida las observaciones son similares y organizar y resumir nuestros datos. 
Tabla V. Índices

\begin{tabular}{|c|c|c|}
\hline Índices & Concepto & Expresión \\
\hline $\begin{array}{c}\text { Índice de } \\
\text { divulgación } \\
\text { por municipio } \\
\text { y dimensión } \\
\text { (IDMD) }\end{array}$ & $\begin{array}{c}\text { Mide el } \\
\text { porcentaje de } \\
\text { divulgación } \\
\text { total de cada } \\
\text { municipio en } \\
\text { cada una de las } \\
\text { dimensiones }\end{array}$ & $I D M D_{j}=\left(\frac{\sum_{i=1}^{M}(A i j)}{M} * p\right) * 100$ \\
\hline $\begin{array}{c}\text { Índice de } \\
\text { divulgación } \\
\text { por municipio } \\
\text { (IDM) }\end{array}$ & $\begin{array}{c}\text { Mide la } \\
\text { divulgación } \\
\text { total de cada } \\
\text { municipio }\end{array}$ & $I D M_{j}=\sum_{i=1}^{D}\left(I D M D_{j}\right)$ \\
\hline $\begin{array}{c}\text { Índice de } \\
\text { divulgación por } \\
\text { ítem (IDI) }\end{array}$ & $\begin{array}{c}\text { Mide el } \\
\text { porcentaje de } \\
\text { municipios que } \\
\text { informan cada } \\
\text { ítem }\end{array}$ & $I D I_{i}=\frac{\sum_{i=1}^{N}(A i j)}{N} * 100$ \\
\hline $\begin{array}{c}\text { Índice de } \\
\text { divulgación } \\
\text { por dimensión } \\
\text { (IDD) }\end{array}$ & $\begin{array}{c}\text { Mide la } \\
\text { divulgación } \\
\text { total de cada } \\
\text { dimensión }\end{array}$ & $I D D_{i}=\left(\frac{\sum_{i=1}^{d}\left(I D I_{i}\right)}{d} * p\right) * 100$ \\
\hline $\begin{array}{c}\text { Mide la } \\
\text { Iivulgación de } \\
\text { total (IDT) }\end{array}$ & $\begin{array}{c}\text { divulgación } \\
\text { total de la } \\
\text { muestra }\end{array}$ & $I D T=\sum_{i=1}^{D}\left(I D D_{i}\right)$ \\
\hline
\end{tabular}

Fuente: Adaptado de Carvalho y otros (2017).

Donde, $M=$ número de ítems que forman cada dimensión; $D=$ número de dimensiones; $\mathrm{A}_{\mathrm{ij}}=$ toma el valor de 1 si la característica que define el indicador (i) está presente en el ayuntamiento (j), y 0 en el caso contrario; $\mathrm{N}=$ número de ayuntamientos; $\mathrm{d}=$ número de ítems de cada dimensión. Al no tener evidencias empíricas sobre la importancia de los distintos índices parciales que componen el índice total se ha asignado el mismo peso específico para cada una de las dimensiones $(p=20 \%)$.

Como primera etapa de la metodología empleamos el método jerárquico de Ward (Ward, 1963). En segundo lugar, aplicamos el método de agrupamiento k-means no jerárquico (Lloyd, 1982; MacQueen, 1967). Esta última metodología se basa en la solución obtenida después de la aplicación del método de Ward. La estimación se realizará mediante el software SPSS en su versión 20.

\section{RESULTADOS Y DISCUSIÓN}

Comenzando por los índices de divulgación por municipio (IDM), observamos que el ayuntamiento que más información divulga es Plasencia, con un índice de divulgación del 62,55\%, seguido de Trujillo $(57,06 \%)$, Malpartida de Cáceres $(42,45 \%)$, Coria $(40,91 \%)$ y Cáceres $(40,44 \%)$. En el otro extremo se sitúan los municipios de Segura de Toro, Casatejada y Santa Cruz de Paniagua, con unos índices de divulgación prácticamente nulos (2\%, $0 \%$ y $0 \%$, respectivamente). Se puede apreciar, además, que tan sólo dos ayuntamientos (Plasencia y Trujillo) presentan unos índices que superan el $50 \%$, más de la mitad de los indicadores propuestos. Por otro lado, si nos fijamos en los índices de divulgación por municipio y dimensión (IDMD), comprobamos que las entidades de La Garganta, Majadas y Pezcueza son las que más información general divulgan, con unos índices del $12 \%$ cada una. Plasencia vuelve a encabezar el ranking con la información que ofrece sobre recursos y apoyo al emprendedor, con un índice del $20 \%$. En relación con el emprendimiento activo, los municipios que más información divulgan son Trujillo y Coria, con unos índices de divulgación del $10 \%$ cada uno. Por tu parte, Trujillo y Malpartida de Cáceres son los que ocupan las primeras posiciones en divulgación de información relativa a emprendimiento digital, con unos índices del $16,67 \%$ cada uno. Por último, vuelven a ser Plasencia, Trujillo y Coria, los que más información ofrecen referente a divulgación y comunicación con el emprendedor (14,55\%, $12,73 \%$ y $10,91 \%$, respectivamente). En cuanto al municipio de Cáceres, capital de la provincia, tan sólo destaca el valor que en él presenta el índice de emprendimiento digital, un $15 \%$, aproximándose a los que más información ofrecen al respecto y superando al municipio de Plasencia, que se encuentra a la cabeza en la mayoría de las dimensiones.

Del mismo modo, se han calculado índices de divulgación por dimensiones (IDD), que miden la divulgación del total de los municipios de la muestra en cada una de las cinco dimensiones y representa la aportación de cada una de ellas al índice de divulgación total (IDT). Se observa, que la información que más peso tiene en el IDT es la relacionada con la información general y el emprendimiento digital, con unos índices del 5,34\% y 4,96\%, respectivamente, mientras que el resto de las dimensiones ofrecen una información prácticamente nula.

Otro aspecto importante del análisis se refiere a los índices de divulgación de información por indicadores (IDI) (Anexo I). En cuanto al análisis de la primera dimensión, información general, se puede observar cómo en la mayoría de los sitios web de los municipios analizados existe un buscador interno que facilita su navegación (93,22\%) y un mapa de la propia web (88,32\%). Asimismo, un $41,82 \%$ de las entidades publica un listado de las empresas del municipio y un $32,71 \%$ contiene links para redes sociales. A pesar de ello, tan sólo un 3,5\% publica información económica clave, como el PIB o la tasa de desempleo; casi un 3\% de los municipios analizados contiene una declaración del máximo responsable sobre la importancia del espíritu empresarial y el desarrollo económico, no incluyendo la mitad de ellos en esta declaración las prioridades y estrategias a alcanzar, así como los logros y fracasos registrados por la entidad; y 
tal sólo un $1 \%$ informa sobre aspectos estratégicos para invertir en esos municipios cacereños.

En segundo lugar, en el análisis de la segunda dimensión, recursos y apoyo al emprendedor, se ha observado que un $30,84 \%$ de los ayuntamientos informa sobre los contactos de interés, siendo éste el ítem más divulgado. Casi un $8 \%$ de los municipios analizados cuenta con un gabinete de apoyo al emprendedor y sólo un 3,04\% informa sobre las formalidades para la creación de empresas y reconoce necesidades específicas de los jóvenes, mujeres y otros grupos destinatarios. Cabe destacar que tan sólo un $2,10 \%$ de los municipios publica en su página web la normativa para la creación de una empresa. Llama la atención que casi ningún ayuntamiento (tan sólo el 1,40\%) hace referencia a la existencia de espacios físicos para el comienzo de una actividad, ni publica información sobre aspectos a seguir para la creación de la misma, y tampoco ofrece soluciones de financiación o informa sobre el coste de iniciar un negocio.

En relación con el emprendimiento activo, se desprende que el $7,01 \%$ de los ayuntamientos ofrece servicios para ayudar a la creación de empresas en el cumplimiento de los requisitos reglamentarios; sólo el 4,91\% difunde información sobre cursos disponibles para desarrollar las habilidades de los empresarios; y casi en el $2 \%$ de los municipios existe un nido de empresas o parque tecnológico municipal y se informa sobre premios o concursos para reconocer públicamente a los empresarios. Como se puede observar, son pocos los ayuntamientos que celebran jornadas de intercambio de experiencias y buenas prácticas y que divulgan programas de educación para el emprendimiento en las escuelas más jóvenes. Además, ningún ayuntamiento tiene tutoriales disponibles para la creación de una empresa ni iniciativas para impulsar la RS de la misma.

Respecto a la cuarta dimensión, emprendimiento digital, se puede observar cómo el $82,48 \%$ de los ayuntamientos posee en su web un buzón para el ciudadano o una sección para quejas y sugerencias. Asimismo, un $34,11 \%$ ofrece la posibilidad de realizar trámites administrativos, permisos y licencias on line y casi el $26 \%$ permite llevar a cabo el seguimiento en el estado de tramitación e incidencias en los procedimientos planteados. Sin embargo, son pocos los ayuntamientos en los que se llevan a cabo campañas de sensibilización y de desarrollo de capacidades para el uso de las TIC, y también son pocos los que facilitan apoyo para la mejora de la tecnología en las pequeñas empresas.

Por último, en la dimensión correspondiente a divulgación y comunicación con el emprendedor, ob- servamos que el $24,53 \%$ de los municipios tiene un espacio para expresar la opinión de los usuarios; alrededor de un $16,12 \%$ dispone de un espacio de satisfacción de los usuarios para el mejoramiento de los gabinetes de apoyo; y un 10,28\% informa sobre las novedades, como nuevos proyectos aprobados o noticias destacadas. Sin embargo, un bajo porcentaje de gobiernos locales publica un boletín municipal con noticias relacionadas con la actividad empresarial (alrededor de un $5 \%$ ) y dispone de un área específica para el emprendedor (3,5\%).

Finalmente, se ha analizado el índice de divulgación total (IDT), que indica que el conjunto de ayuntamientos de la provincia de Cáceres analizados divulga de media el $12,86 \%$ de la información total sobre emprendimiento. Podemos decir, por tanto, que la divulgación de información que se ofrece es escasa. Sin embargo, no distan mucho de los obtenidos en otros estudios sobre divulgación de información, como los de Nevado y otros (2013), en los que se evidencia la existencia de un grado de divulgación de información sobre RS de un $19,19 \%$, en una muestra de municipios extremeños. Con estos resultados pretendemos, por un lado, dar a conocer las carencias existentes en relación con la información que ofrecen los gobiernos locales cacereños a los emprendedores a través de sus sitios web, y, por otro, alentarles en su utilización como medio de comunicación de la información sobre emprendimiento.

En relación con el segundo de nuestros objetivos, clasificar los ayuntamientos en función de las semejanzas y diferencias respecto a los niveles de divulgación sobre emprendimiento, se ha llevado a cabo un Análisis Cluster (Vilà y otros, 2014; Rubio y Vilà, 2017). En un primer momento se procede a aplicar el análisis cluster no jerárquico K-medias (Quick Cluster) (Tabla VI) empleando los índices de divulgación previamente diseñados y valorados para la muestra de ayuntamientos cacereños objeto de estudio. Para fijar a priori el número de grupos a determinar y extraer o formar en el Quick Cluster, se utiliza el método jerárquico de Ward. En el dendograma (Anexo II) se puede apreciar cómo se forman los conglomerados en cada paso y es posible evaluar los niveles de similitud (o distancia) de los conglomerados formados. Detectamos dos agrupaciones con el mayor nivel de similitud posible, y un pequeño número de ayuntamientos aislados. Por ello, concluimos que la formación jerárquica de conglomerados de casos en función de las variables utilizadas (los 6 indicadores de divulgación) corresponde a 3 agrupaciones, una de las cuales tan sólo incluye cinco municipios aparentemente incapaces de reagruparse con el resto, que se consideran "outlier". 
Tabla VI. Resultados del Quick Cluster con los seis índices de divulgación diseñados. Número de casos por conglomerado

\begin{tabular}{|l|l|r|}
\hline \multirow{2}{*}{ Conglomerado } & $\mathbf{1}$ & 54,000 \\
\cline { 2 - 3 } & $\mathbf{2}$ & 5,000 \\
\cline { 2 - 3 } & $\mathbf{3}$ & 154,000 \\
\hline Válidos & & 213,000 \\
\hline Perdidos & 9,000 \\
\hline
\end{tabular}

Los resultados del Análisis de la Varianza (Tabla VII) demuestran que, a un nivel de confianza de más del 95\%, todos los índices de divulgación resultan significativos, lo que manifiesta que todos ellos están contribuyendo de igual manera a la formación de los tres grupos. Esto indica que los tres grupos obtenidos son muy discriminantes, es decir, presentan comportamientos distintos entre sí.

Podemos concluir, por tanto, que, de forma estática y para nuestra muestra de ayuntamientos españoles pertenecientes a la provincia de Cáceres, el empleo conjunto de los cinco subíndices de divulgación diseñados y del índice general nos ha permitido diferenciar claramente agrupaciones por nivel de divulgación.

El estadístico descriptivo media de cada una de las variables que resultaron significativas, se muestra en la Tabla VIII. Dicho estadístico representa el valor medio que toma cada índice de divulgación en cada uno de los tres conglomerados obtenidos y pone de manifiesto los diferentes niveles de divulgación de información dirigida al emprendedor en nuestra muestra de ayuntamien- tos de la provincia de Cáceres. Así, se pueden distinguir 54 ayuntamientos que divulgan más información relativa a emprendimiento en sus sitios web (cluster 1) y 154 ayuntamientos que divulgan menos información relativa a emprendimiento en sus webs (cluster 3 ). El cluster 2, que contiene únicamente cinco ayuntamientos, constituye un valor extremo (outlier) que excluimos de la muestra por los valores medios que toman en él las distintas variables (índices de divulgación) analizadas. Por tanto, la muestra definitiva pasa a componerse de 208 ayuntamientos.

En cuanto a las características poblacionales, socio-económicas, fiscales y políticas que los ayuntamientos presentan en cada uno de estos clusters, en general, se observa que los valores medios de las variables explicativas cuantitativas son similares en ambas agrupaciones. No obstante, el cluster de ayuntamientos que más divulga (cluster 1 ) se caracteriza por poseer un número de habitantes medio superior (1.500 frente a 1.016 en el cluster 3), un total de gastos medios superior (1.200.000€ frente a $900.000 €$ en el cluster $3)$, mayor deuda pública media (180.000€ frente a $158.000 €$ en el cluster 3), mayor tasa de paro $(0,24$ frente a 0,23 en el cluster 3$)$, y un número de partidos políticos superior (2,7 frente a 2,55 en el cluster 3). Por lo que respecta a las variables explicativas cualitativas, en ambos cluster existe una estabilidad política considerable (casi $80 \%$ de mayoría absoluta), y predomina el sexo masculino y la presencia del PSOE en la alcaldía (más en el caso del clúster 3 ). Las Tablas IX y X muestran los valores medios de las variables explicativas cualitativas y cuantitativas en cada uno de los dos cluster indentificados.

Tabla VII. Análisis de la Varianza

\begin{tabular}{|c|c|c|c|c|c|c|}
\hline & \multicolumn{2}{|c|}{ Conglomerado } & \multicolumn{2}{c|}{ Error } & \\
\hline & $\begin{array}{c}\text { Media } \\
\text { cuadrática }\end{array}$ & gl. & $\begin{array}{c}\text { Media } \\
\text { cuadrática }\end{array}$ & gl. & F & Sig. \\
\hline IDA & 5580,683 & 2 & 16,699 & 210 & 334,198 & 0,000 \\
\hline IDD1 & 21,617 & 2 & 3,613 & 210 & 5,983 & 0,003 \\
\hline IDD2 & 38,433 & 2 & 3,303 & 210 & 11,634 & 0,000 \\
\hline IDD3 & 15,884 & 2 & 2,068 & 210 & 7,682 & 0,001 \\
\hline IDD4 & 322,489 & 2 & 10,579 & 210 & 30,485 & 0,000 \\
\hline IDD5 & 75,710 & 2 & 4,663 & 210 & 16,236 & 0,000 \\
\hline
\end{tabular}


Tabla VIII. Resultados del Quick Cluster con todos los índices de divulgación. Centros de los conglomerados finales

\begin{tabular}{|c|c|c|c|}
\hline & \multicolumn{3}{|c|}{ Conglomerado } \\
\hline & $1(54)$ & $2(5)$ & $3(154)$ \\
\hline IDA & 19,97 & 48,68 & 9,24 \\
\hline IDD1 & 6,11 & 5,40 & 5,07 \\
\hline IDD2 & 1,93 & 0,33 & 0,56 \\
\hline IDD3 & 1,04 & 0,00 & 0,16 \\
\hline IDD4 & 7,93 & 5,33 & 3,92 \\
\hline IDD5 & 2,74 & 0,73 & 0,81 \\
\hline
\end{tabular}

Tabla IX. Estadísticos descriptivos CLUSTER 1

\begin{tabular}{|l|c|c|c|c|c|}
\hline & N & Mínimo & Máximo & Media & Desv. típ. \\
\hline N. Habitantes & 54 & 90 & 17247 & 1470,39 & 2508,469 \\
\hline Total gastos & 54 & 0,00 & 11041875,10 & 1244791,6324 & 1814951,81661 \\
\hline Deuda pública & 54 & 0,00 & 4885577,68 & 183721,5035 & 708145,90984 \\
\hline Población dependiente & 54 & 0 & 1 & 0,43 & 0,051 \\
\hline Tasa de paro & 54 & 0,0928 & 0,4918 & 0,242596 & 0,0720557 \\
\hline $\begin{array}{l}\text { N. de partidos que } \\
\text { intervienen en las elecciones } \\
\text { (Competencia política) }\end{array}$ & 54 & 2 & 7 & 2,70 & 0,964 \\
\hline Tasa de abstención & 54 & 0,0608 & 0,3559 & 0,164711 & 0,0562585 \\
\hline N válido (según lista) & 54 & & & & \\
\hline
\end{tabular}

Tabla X. Estadísticos descriptivos CLUSTER 3

\begin{tabular}{|l|c|c|c|c|c|}
\hline & N & Mínimo & Máximo & Media & Desv. típ. \\
\hline N. Habitantes & 154 & 51 & 9879 & 1016,49 & 1401,405 \\
\hline Total gastos & 154 & 0,00 & 8308804,05 & 905660,0215 & 1188092,52485 \\
\hline Deuda pública & 154 & 0,00 & 3325590,02 & 158373,0996 & 425768,05722 \\
\hline Población dependiente & 154 & 0 & 1 & 0,43 & 0,051 \\
\hline Tasa de paro & 154 & 0,0672 & 0,4512 & 0,230875 & 0,0643086 \\
\hline $\begin{array}{l}\text { N. de partidos que } \\
\text { intervienen en las elecciones } \\
\text { (Competencia política) }\end{array}$ & 154 & 2 & 7 & 2,55 & 0,768 \\
\hline Tasa de abstención & 154 & 0,0000 & 0,4234 & 0,166808 & 0,0620697 \\
\hline N válido (según lista) & 154 & & & & \\
\hline
\end{tabular}

\section{CONCLUSIONES, IMPLICACIONES Y FUTURAS LÍNEAS DE INTERÉS}

Este estudio analiza el grado y el tipo de información sobre emprendimiento que los municipios cacereños divulgan a través de sus sitios web, e identifica la existencia de dos categorías de ayuntamientos en función del nivel de divulgación y las características que éstos presentan en cada una de las agrupaciones.
Los resultados revelan la existencia en las webs de este tipo de información. Sin embargo, como se ha podido comprobar a través del índice total de divulgación (ITD), la información que se divulga es escasa $(12,86 \%)$. Estas carencias ponen de manifiesto la necesidad de ofrecer oportunidades de mejora en los sistemas de información de cara a la comunicación con los emprendedores. Destaca especialmente el compromiso que muestra el ayuntamiento de Plasencia, ya que es el que más 
información divulga de toda la provincia de Cáceres destinada a estimular la creación de empresas innovadoras que contribuyan a acelerar el crecimiento económico y el desarrollo sostenible en el territorio. Plasencia es la ciudad más poblada del norte de la región extremeña, con un rico patrimonio monumental y natural, y está rodeada por poblaciones de cinco mancomunidades diferentes, por lo que ofrece un amplio conjunto de servicios del gobierno extremeño y del gobierno nacional para su población y para la población del territorio que la rodea. Todo ello, unido al desarrollo en infraestructura que ha experimentado en los últimos años, la convierten en foco de aceleración económica y de empleo que el propio municipio está contribuyendo a dinamizar mediante el uso de las TICs. Los ayuntamientos que más información divulgan son, además de Plasencia, Trujillo y Malpartida de Cáceres mientras que Segura de Toro, Casatejada y Santa Cruz de Paniagua son los que menos información ofrecen.

Por lo que respecta a las cinco dimensiones que abarca nuestro ITD, destaca la difusión de información relacionada con aspectos generales, y emprendimiento digital y comunicación con el emprendedor. Llama la atención la escasa información que se ofrece sobre recursos y apoyo al emprendedor y emprendimiento activo.

Cabe destacar el modesto valor que presentan los índices individuales para Cáceres capital, en general, pero sobre todo el índice relativo a la dimensión mencionada "Recursos y apoyo al emprendedor" (Dimensión 2), un 4,17\%. A este respecto, llama la atención el hecho de que, a pesar de su extensión, tan sólo cuente con dos puntos de acompañamiento empresarial, como se ha señalado anteriormente. Bien es cierto que su ubicación no es tan estratégica como la de Plasencia, que suministra servicios públicos a un buen número de poblaciones de diferentes mancomunidades que la rodean. Es por ello que Plasencia presenta un índice de divulgación sobre esta dimensión, relacionada con información sobre permisos y normativas, opciones de financiación, ayudas

\section{NOTAS}

1. Cuando se habla de información voluntaria se está haciendo referencia a la información estratégica, consecución de objetivos, proyectos de expansión, planes anuales, descripción del entorno competitivo o información sobre procesos productivos, entre otras. económicas públicas, posibilidad de expansión a nuevos mercados, etc., del $20 \%$.

En relación con las agrupaciones identificadas, existen dos categorías de ayuntamientos que presentan comportamientos distintos entre sí en cuanto al nivel de divulgación, cuyo tamaño está inversamente relacionado con la divulgación de información dirigida al emprendedor. El grupo de ayuntamiento que más divulga se caracteriza por estar más poblado, poseer, en términos medios, un mayor nivel de gasto y deuda pública, y una tasa de paro superior al grupo de ayuntamientos menos comprometido con la divulgación de información de apoyo al emprendimiento. Los resultados obtenidos corroboran la existencia de una implicación, aunque aún modesta, de las administraciones públicas locales de la provincia de Cáceres en relación con la promoción del emprendimiento y los ecosistemas empresariales, sobre todo en aquellos municipios con una mayor tasa de paro. Precisamente la elevada tasa de paro de la región nos llevó a seleccionarla como población objeto de estudio. Cabría ahora plantearse analizar la medida en que estas actuaciones de los ayuntamientos contribuyen a revertir la tendencia en la tasa de paro.

En cuanto a las implicaciones de los resultados obtenidos, podemos enfatizar que, en el ámbito académico, nuestra investigación contribuye a la literatura existente con una mayor explicación de lo que está sucediendo en relación con la divulgación de información sobre emprendimiento en el sector público, debido a la escasez de trabajos en esta materia.

La principal limitación de este estudio es su carácter meramente descriptivo, ya que utiliza datos de corte transversal que no permiten establecer inferencias. Se propone, por tanto, una interesante línea de trabajo enfocada al análisis de los factores explicativos del desarrollo de estas prácticas de divulgación, considerando su evolución en el tiempo y evidenciando su contribución al desarrollo económico y a la creación de empleo en el territorio objeto de estudio a través del emprendimiento.

2. Más información disponible en: https://transparencia. org.es/indice-de-los-ayuntamientos-ita/. Consultada a fecha $11 / 04 / 2018$ 


\section{REFERENCIAS}

Abela, J. A. (2000). Las técnicas de análisis de contenido: una revisión actualizada. Documentos de Trabajo. Serie Sociología, 10 (2), 1-34. Fundación Centro Estudios Andaluces. Universidad de Granada. Disponible en: http://www.albertomayol.cl/wp-content/uploads/2014/08/Andreu-Analisis-de-contenido.pdf [Fecha de consulta: 20/05/2018].

Alcaraz-Quiles, F. J.; Navarro-Galera, A.; Ortiz-Rodríguez, D. (2015). Factors determining online sustainability reporting by local governments. International Review of Administrative Sciences, 81 (1), 79-109. https:// doi.org/10.1177/0020852314541564

Baptista, M. (2005). E-government and state reform: Policy dilemmas for Europe. The Electronic Journal of e-Government, 3(4), 167-174.

Batalli, M. (2011). Impact of Public Administration Innovations on Enhancing the Citizens' Expectations. International Journal of e-Education, e-Business, e-Management and e-Learning, 1(2), 156-162.

Bekkers, V.; Van Duivenboden, H.; Thaens, M. (2006). Public Innovation and Communication Technology: Relevant Backgrounds and Concepts. En: V. Bekkers, $\mathrm{H}$. van Duivenboden and M. Thaens (eds.), Information and Communication Technology and Public Innovation, pp.3-21. Amsterdam: Assessing the ICT-Driven Modernization of Public Administration, IOS Press.

Beuren, I. M.; Angonese, R. (2015). Instruments for determining the disclosure index of accounting information. Revista Eletrônica de Estratégia e Negócios - REEN, 8(1), 120-144. https://doi.org/10.19177/ reen.v8e12015120-144

Bonsón, E.; Escobar, T. (2004). La difusión voluntaria de información financiera en Internet. Un análisis comparativo entre Estados Unidos, Europa del Este y la Unión Europea. Spanish Journal of Finance and Accounting/Revista Española de Financiación y Contabilidad, 33(123), 1063-1101. https://doi.org/10.1080/02102412.2004.10779539

Brusca, I.; Manes Rossi, F.; Aversano, N. (2016). Online sustainability information in Local Governments in an austerity context: an empirical analysis in Italy and Spain. Online Information Review, 40(4), 497-514. https://doi.org/10.1108/OIR-05-2015-0161

Caba, C.; López, A. M.; Rodríguez, M. P. (2005). Citizens' access to on-line governmental financial information: Practices in the European Union Countries. Government Information Quarterly, 22 (2), 258-276. https://doi. org/10.1016/j.giq.2005.02.002

Caba, C. ; Rodríguez, M. ; López, A. M. (2008). e-Government process and incentives for online public financial information. Online Information Review, 32 (3), 379400. https://doi.org/10.1108/14684520810889682

Cárcaba, A.; García, J. (2008). Determinantes de la divulgación de información contable a través de Internet por parte de los gobiernos locales. Revista Española de Financiación y Contabilidad, 37 (137), 63-84. https:// doi.org/10.1080/02102412.2008.10779639
Cárcaba, A.; García, J. (2010). Determinants of online reporting of accounting Information by Spanish Local Government authorities. Local Government Studies, 36(5), 679-695. https://doi.org/10.1080/03003930.2 010.506980

Carvalho, L.; Gallardo, V. D.; Nevado, G. M. T. (2017). Local municipalities' involvement in promoting entrepreneurship: An analysis of web page orientation to the entrepreneurs in Portuguese municipalities. En: Carvalho, L. (ed.), Handbook of Research on Entrepreneurial Ecosystems and Social Dynamics in a Globalized World, pp.1-19. Évora, Portugal: IGI Global. https://doi.org/10.4018/978-1-4666-8216-0.ch009

Casasola, L. A.; Guerra, J. C.; Casasola, M. A.; Pérez, V. A. (2017). La accesibilidad de los portales web de las universidades públicas andaluzas. Revista Española de Documentación Científica, 40(2), e169. https://doi. org/10.3989/redc.2017.2.1372

Cronbach, L. J. (1951). Coefficient alpha and the internal structure of tests. Psychometrika, 16 (3), 297-334. https://doi.org/10.1007/BF02310555

Cuadrado-Ballesteros. B. (2014). The impact of functional decentralization and externalization on local government transparency. Government Information Quarterly, 31 (2), 265-277. https://doi.org/10.1016/j. giq.2013.10.012

Cueto, C.; Cuesta, M. de la; Moneva, J. M. (2014). La oferta informativa sobre Responsabilidad Social Corporativa en las administraciones locales españolas. Prisma Social: Revista de investigación social, (12), 646-687.

Díaz, C. V. (2009). Transparencia de la información económico-financiera a través del e-Gobierno o Gobierno Electrónico: Caso Español. Perspectivas, (24), 59-90.

Dunleavy, P.; Margetts, H.; Bastow, S.; Tinkler, J. (2006). Digital era governance, IT corporations, the state and the e-government, Oxford University Press. https://doi. org/10.1093/acprof:oso/9780199296194.001.0001

Farneti, F.; Guthrie, J. (2009). Sustainability Reporting by Australian Public Sector Organizations. Accounting Forum, 33(2), 89-98. https://doi.org/10.1016/j.accfor.2009.04.002

Ferreira, G. A. ; Barros, R. de (2011). Acessibilidade dos deficientes visuais e cegos às informações de bibliotecas universitárias na web. Informação \& Sociedade, 21(2), 151-163.

Frías-Aceituno, J. V.; Marques, M. D. C.; Rodríguez-Ariza, L. (2013). Divulgación de información sostenible: ¿se adapta a las expectativas de la sociedad? Spanish Accounting Review/ Revista de Contabilidad, 16(2), 147-158. https://doi.org/10.1016/j.rcsar.2013.07.004

Gandía, J. L.; Archidona, M. C. (2008). Determinants of web site information by Spanish city councils. Online Information Review, 32(1), 35-57. https://doi. org/10.1108/14684520810865976 
García-Sánchez, I.M.; Frías-Aceituno, J.V.; RodríguezDomínguez, L. (2013). Determinants of corporate social disclosure in Spanish Local governments. Journal of Cleaner Production, 39, 60-72. https://doi. org/10.1016/j.jclepro.2012.08.037

George, D.; Mallery, P. (2003). Using SPSS for Windows step by step: A Simple Guide and Reference (4. a ed.). Boston: Allyn \& Bacon.

González, J. A. M.; Vergueiro, W. D. C. S.; Sonia, S. C. (2012). Análise do contexto de emprego dos profissionais brasileiros da Informação-Documentação a partir de ofertas de trabalho na Web feitas por empresas e instituições. Informação \& Sociedade, 22(1), 67-78.

Gutiérrez-Ponce, H.; Creixans-Tenas, J.; Arimany-Serrat, N. (2018). Características de las páginas webs de los hospitales de la comunidad de Madrid: relación entre la calidad web y la responsabilidad social corporativa. Revista Española de Documentación Científica, 41(1), e194. https://doi.org/10.3989/redc.2018.1.1465

Hashim, F.; Amran, A.; Nejati, M.; Ismail, Z. (2016). Examining the determinants of sustainability web-reporting by local authorities in Malaysia. International Journal of Society Systems Science, 8 (2), 155-170. https://doi.org/10.1504/IJSSS.2016.077012

Hilera, J.R.; Fernández, L.; Suárez, E.; Vilar, E.T. (2013). Evaluación de la accesibilidad de páginas web de universidades españolas y extranjeras incluidas en rankings universitarios internacionales. Revista Española de Documentación Científica, 36(1), e004. https://doi. org/10.3989/redc.2013.1.913

INE (2017). Encuesta de población activa. Disponible en: http://www.ine.es/dyngs/INEbase/es/operacion.htm?c= Estadistica_C\&cid $=1254736176918 \&$ menu $=$ ultiDatos\&i $\mathrm{dp}=1254735976595$ [Fecha de consulta: 25/09/2017].

Jain, A. K. (2010). Data clustering: 50 years beyond K-means. Pattern Recognistion Letters, 31 (8), 651666. https://doi.org/10.1016/j.patrec.2009.09.011

Jobson, J.D. (1992). Applied Multivariate Data Analysis. Volume II: Categorical and Multivariate Methods. New York: Editorial Springer-Verlag. https://doi. org/10.1007/978-1-4612-0921-8

Jorge, S.; Sá, P.M.; Pattaro, A.F.; Lourenço, R. (2011). Local Government financial transparency in Portugal and Italy: a comparative exploratory study on its determinants. En: 13th Biennial CIGAR conference, bridging public sector and non-profit sector accounting, pp. 9-10. Ghent, Belgium.

Joseph, C.; Taplin, R. (2011). The measurement of sustainability disclosure: Abundance versus occurrence. Accounting Forum, 35 (1), 19-31. https:// doi.org/10.1016/j.accfor.2010.11.002

Krippendorff, K. (1990). Metodología de análisis de contenido. Teoría y Práctica. Barcelona: Ediciones Paidós Ibérica.

Krippendorff, K. (2004). Reliability in Content Analysis. Human Communication Research, 30(3), 411-433. https://doi.org/10.1111/j.1468-2958.2004.tb00738.x
Laswad, F.; Fisher, R.; Oyelere, P. (2005). Determinants of voluntary Internet financial reporting by local government authorities. Journal of Accounting and Public Policy, 25, 101-121. https://doi.org/10.1016/j. jaccpubpol.2004.12.006

Lee, J.H.; Venkataraman, S. (2006). Aspirations, market offerings, and the pursuit of entrepreneurial opportunities. Journal of Business Venturing, 21, 107123. https://doi.org/10.1016/j.jbusvent.2005.01.002

Lloyd, S. (1982). Least squares quantization in PCM. IEEE Transactions on Information Theory, 28 (2),129-137. https://doi.org/10.1109/TIT.1982.1056489

Lodhia, S.; Jacobs, K.; Park, Y. J. (2012). Driving public sector environmental reporting: the disclosure practices of Australian commonwealth departments. Public Management Review, 14(5), 631-647. https:// doi.org/10.1080/14719037.2011.642565

Lombard, M.; Snyder-Duch, J.; Bracken, C. C. (2002). Content Analysis in Mass Communication: Assessment and Reporting of Intercoder Reliability. Human Communication Research, 28(4), 587-604. https:// doi.org/10.1111/j.1468-2958.2002.tb00826.x

MacQueen, J. (1967). Some methods for classification and analysis of multivariate observations. En: Fifth Berkeley Symposium on Mathematics. Statistics and Probability, pp. 281-297. Los Ángeles: University of California Press.

Martínez, R.; Berrocal, S. (2017). Museos y engagement. La calidad de los espacios web como soporte del compromiso. Revista Española de Documentación Científica, 40(1), e166. https://doi.org/10.3989/ redc.2017.1.1383

Mendes, H. C.; Santos, C.; Ferreira, A. D. C.; Marques, R. P.; Carmo, G. M. do; Silva, J. O. Da. (2016). Local authorities and the disclosure of financial information via the internet: The Portuguese Case. En: Global Perspectives on Risk Management and Accounting in the Public Sector, pp. 274-295. IGI Global. https://doi. org/10.4018/978-1-4666-9803-1.ch014

Minniti, M. (2004). Entrepreneurial alertness and asymmetric information in a spin-glass model. Journal of Business Venturing, 19(5), 637-658. https://doi. org/10.1016/j.jbusvent.2003.09.003

Mir, M. Z.; Chatterjee, B.; Taplin, R. (2015). Political competition and environmental reporting: Evidence from New Zealand Local Governments. Asian Review of Accounting, 23(1), 17-38. https://doi.org/10.1108/ ARA-02-2014-0027

Moneva, J.; Martín, E. (2012). Universidad y Desarrollo sostenible: Análisis de la rendición de cuentas de las universidades públicas desde un enfoque de responsabilidad social. Revista Iberoamericana de Contabilidad de Gestión, 10 (19), 1-18.

Navarro, G. A.; Alcaraz, F. J.; Ortiz, D. (2010). La divulgación de información sobre responsabilidad corporativa en administraciones públicas: un estudio empírico en gobiernos locales. Revista de Contabilidad, 
13 (2), 285-314. https://doi.org/10.1016/S11384891(10)70019-4

Navarro, G. A. ; Tirado V. P.; Ruiz, M.; Ríos, A. de los (2015). Divulgación de información sobre responsabilidad social de los gobiernos locales europeos: El caso de los países nórdicos. Gestión y Política Pública, 24 (1), 229-269.

Navarro, G. A.; Alcaraz, F. J.; Ortiz, D. (2016). Online dissemination of information on sustainability in regional governments. Effects of technological factors. Government Information Quarterly, 33 (1), 53-66. https://doi.org/10.1016/j.giq.2015.12.003

Nevado, M. T.; Gallardo, D.; Sánchez, M. I. (2013). La administración local y su implicación en la creación de una cultura socialmente responsable. Prisma Social: Revista de investigación social, 10, 64-118.

Nevado, M. T.; Gallardo, D. (2016). Información sobre Responsabilidad Social contenida en las páginas web de los ayuntamientos. Estudio en la región del Alentejo. Revista Española de Documentación Científica, 39(4), e150. https://doi.org/10.3989/redc.2016.4.1353

Oates, G.; Moradi-Motlagh, A. (2016). Is voluntary disclosure of environmental performance associated with actual environmental performance? Evidence from Victorian Local Governments, Australia. Australasian Journal of Environmental Management, 23 (2), 194205. https://doi.org/10.1080/14486563.2015.1082156

OECD (1999). Government of the Future: Getting From Here to There. París: OECD.

OSI y LGPSRI (2007). ICT for Local Government HANDBOOK. E-Governance Academy. Disponible en: http://ega.ee/wp-content/uploads/2015/02/project_ ICT_for_Local_Government.pdf [Fecha de consulta: 20/02/2017].

Peña, M. (2012). La importancia del acuerdo entre codificadores para el análisis de contenido. Comunicación y medios, 25,47-56.

Pereira, A. S.; Machado, A. M.; Carneiro, T. C. J. (2013). Avaliação da acessibilidade dos sítios eletrônicos das instituições de ensino superior brasileiras. Informação \& Sociedade, 23(3), 123-142.

Piñeiro; V.; Igartua, J. J.; Marañón, F. (2017). El diseño de las sedes web municipales de España. Una propuesta metodológica para su análisis. Revista Española de Documentación Científica, 40(1), e164. https://doi. org/10.3989/redc.2017.1.1368

Prado-Lorenzo, J.M.; García-Sánchez, I.M.; CuadradoBallesteros, B. (2012). Sustainable cities: dopolitical factors determine the quality of life? Journal of Cleaner Production, 21(1), 34-44. https://doi.org/10.1016/j. jclepro.2011.08.021

Rodríguez, M. P.; Caba, C.; López, A. M. (2006). Cultural contexts and governmental digital reporting. International Review of Administrative Sciences, 72 (2), 269-290. https://doi.org/10.1177/0020852306064614

Rodríguez, M. P.; Caba, C.; López, A. M. (2007). E-government and public financial reporting. The case of Spanish regional governments. The American Review of Public Administration, 37 (2), 142-177. https://doi.org/10.1177/0275074006293193

Rodríguez, D. L.; Gallego, A. I.; García, S. I. M. (2010). Determinantes de la divulgación voluntaria de información estratégica en internet: un estudio de las empresas cotizadas. Revista Europea de Dirección y Economía de la Empresa, 19 (1), 9-26.

Rubio, M. J.; Vilà, R. (2017). El análisis de conglomerados bietápico o en dos fases con SPSS. REIRE. Revista d'Innovació i Recerca en Educació, 10 (1), 118-126. https://doi.org/10.1344/reire2017.10.11017

Serrano, C.; Rueda, M.; Portillo, P. (2009). Factors influencing e-disclosure in local public administrations. Environment and Planning C-Government and Policy, 27 (2), 355-378. https://doi.org/10.1068/ c07116r

Stiglitz, J.E. (2000). The contributions of the economics of information to twentieth century economics. Quarterly Journal of Economics, 115, 1441-1478. https://doi. org/10.1162/003355300555015

Sullivan, D. M.; Meek, W. R. (2012). Gender y entrepreneurship: A review y process model. Journal of Managerial Psychology, 27 (5), 428-458. https:// doi.org/10.1108/02683941211235373

Vilà, R.; Rubio, M. J.; Berlanga, V. ; Torrado, M. (2014). Com aplicar un clúster jeràrquic en SPSS. REIRE. Revista d'Innovació i Recerca en Educació, 7(1), 113127. http://dx.doi.org/10.1344/reire2014.7.1717

Ward, J. H. Jr. (1963). Hierarchical grouping to optimize an objective function. Journal of the American Statistical Association, 58 (301), 236-244. https://doi.org/10.10 80/01621459.1963.10500845

Yu, H. (2010). On the determinants of internet-based disclosure of government financial information. En: International Conference on Management and Service Science (MASS). IEEE. 


\section{ANEXOS}

Anexo I. Índices de divulgación por indicadores (IDI)

\begin{tabular}{|l|c|}
\hline DIMENSIÓN 1: INFORMACIÓN GENERAL & IDI \\
\hline $\begin{array}{l}\text { 1.1. Existe una declaración del máximo responsable sobre la importancia del espíritu empresarial y el } \\
\text { desarrollo económico }\end{array}$ & 2,80 \\
\hline 1.2. Se incluyen en esta declaración prioridades y estrategias a alcanzar & 1,40 \\
\hline 1.3. Se incluyen eventos, logros y fracasos registrados por la entidad & 1,40 \\
\hline 1.4. Existe un mapa de la propia web & 88,32 \\
\hline 1.5. Existe un buscador interno para facilitar su navegación & 93,22 \\
\hline 1.6. Existe la posibilidad de escuchar la página & 0,47 \\
\hline 1.7. Existen links para redes sociales & 32,71 \\
\hline 1.8. Existe un listado de empresas del territorio & 41,82 \\
\hline 1.9. Se publica información económica clave, como el PIB o la tasa de desempleo & 3,50 \\
\hline 1.10. Se informa sobre los aspectos estratégicos o razones para invertir en ese municipio & 1,17 \\
\hline
\end{tabular}

\begin{tabular}{|l|c|}
\hline DIMENSIÓN 2: RECURSOS Y APOYO AL EMPRENDEDOR & IDI \\
\hline 2.1. Existe algún gabinete u órgano de apoyo al emprendedor & 7,71 \\
\hline 2.2. Está disponible la normativa para la creación de una empresa & 2,10 \\
\hline 2.3. Se hace referencia a la disponibilización de algún espacio físico para el comienzo de la actividad & 1,40 \\
\hline 2.4. Se publica información sobre los procedimientos a seguir para la creación de un negocio & 1,87 \\
\hline 2.5. Existen objetivos específicos u objetivos medibles para aumentar la actividad empresarial & 0,47 \\
\hline $\begin{array}{l}\text { 2.6. Se difunde información sobre la iniciativa empresarial, incluido el emprendimiento social y su impacto } \\
\text { en la economía }\end{array}$ & 0,93 \\
\hline $\begin{array}{l}\text { 2.7. Se informa sobre los contactos de interés } \\
\text { 2.8. Se reconocen necesidades específicas de los jóvenes, mujeres y otros grupos destinatarios }\end{array}$ & 30,84 \\
\hline $\begin{array}{l}\text { 2.9. Se informa sobre los incentivos fiscales de la inversión (soluciones de financiación de capital de } \\
\text { riesgo) }\end{array}$ & 3,04 \\
\hline 2.10. Existe información sobre protección de conocimiento, marca etc. & 1,17 \\
\hline 2.11. Se informa sobre formalidades para la creación de empresas & 0,47 \\
\hline 2.12. Se hace referencia al tiempo y coste de iniciar un negocio & 3,04 \\
\hline
\end{tabular}

\begin{tabular}{|l|c|}
\hline DIMENSIÓN 3: EMPRENDIMIENTO ACTIVO & IDI \\
\hline $\begin{array}{l}\text { 3.1. Existen mecanismos, como foros de múltiples partes interesadas para promover el diálogo sobre la } \\
\text { iniciativa empresarial }\end{array}$ & 0,47 \\
\hline 3.2. Hay tutoriales disponibles de ayuda para la creación de una empresa & 0,00 \\
\hline 3.3. Existen cursos disponibles para desarrollar las habilidades de los empresarios & 4,91 \\
\hline 3.4. Existen concursos, premios o eventos similares para reconocer públicamente a los empresarios & 1,87 \\
\hline $\begin{array}{l}\text { 3.5. Ofrece el municipio servicios para ayudar a la creación de empresas en el cumplimiento de los } \\
\text { requisitos reglamentarios }\end{array}$ & 7,01 \\
\hline 3.6. Hay claridad sobre las prioridades y el tipo de emprendimiento que el municipio quiere animar & 0,70 \\
\hline 3.7. Se celebran jornadas de intercambio de experiencias y buenas prácticas & 1,17 \\
\hline 3.8. Existen programas de educación para el emprendimiento en las escuelas más jóvenes & 0,70 \\
\hline 3.9. Existe una incubadora, nido de empresas o parque tecnológico municipal & 1,87 \\
\hline 3.10. Existen iniciativas para alentar la RS de las empresas & 0,00 \\
\hline
\end{tabular}




\begin{tabular}{|l|c|}
\hline DIMENSIÓN 4: EMPRENDIMIENTO DIGITAL & IDI \\
\hline $\begin{array}{l}\text { 4.1. Existen campañas de sensibilización y de desarrollo de capacidades en el uso de las TIC y economía } \\
\text { digital }\end{array}$ & 2,34 \\
\hline $\begin{array}{l}\text { 4.2. Facilita el municipio apoyo para la mejora de la tecnología en las pequeñas empresas } \\
\text { 4.3. Posibilidad de realizar trámites administrativos, permisos, licencias on line }\end{array}$ & 34,11 \\
\hline $\begin{array}{l}\text { 4.4. Seguimiento on line del estado de tramitación e incidencias del procedimiento planteado } \\
\text { 4.5. Existe un buzón ciudadano o una sección para quejas, sugerencias }\end{array}$ & 82,48 \\
\hline $\begin{array}{l}\text { 4.6. Existe una plataforma para el intercambio empresarial, portales empresariales, ferias, asociaciones y } \\
\text { clubes empresariales }\end{array}$ & 1,17 \\
\hline
\end{tabular}

\begin{tabular}{|c|c|}
\hline DIMENSIÓN 5: DIVULGACIÓN Y COMUNICACIÓN CON EL EMPRENDEDOR & IDI \\
\hline 5.1. Existe un área específica para el emprendedor & 3,50 \\
\hline 5.2. Está accesible en la web y fácil de identificar & 3,97 \\
\hline 5.3. Se divulgan las oportunidades de inversión & 1,40 \\
\hline 5.4. Se informa sobre las novedades como nuevos proyectos aprobados, noticias destacadas... & 10,28 \\
\hline 5.5. Existe un espacio de satisfacción de los usuarios para el mejoramiento de los gabinetes de apoyo & 16,12 \\
\hline 5.6. Existe un espacio para expresar las opiniones de los usuarios & 24,53 \\
\hline 5.7. Se publica un Boletín Municipal con noticias relacionadas con la actividad empresarial & 4,67 \\
\hline 5.8. Se informa sobre lo necesario para iniciar una actividad & 1,64 \\
\hline 5.9. Se difunde o se da publicidad a las nuevas empresas creadas recientemente & 2,34 \\
\hline 5.10. Existe ligación a la universidad o al instituto de enseñanza superior & 1,17 \\
\hline 5.11. Se llevan a cabo campañas para promover el espíritu empresarial & 1,40 \\
\hline
\end{tabular}

Anexo II. Dendograma

$$
\text { H I E R A R C H I C A L C L U S T E R A N A L Y S I S }
$$

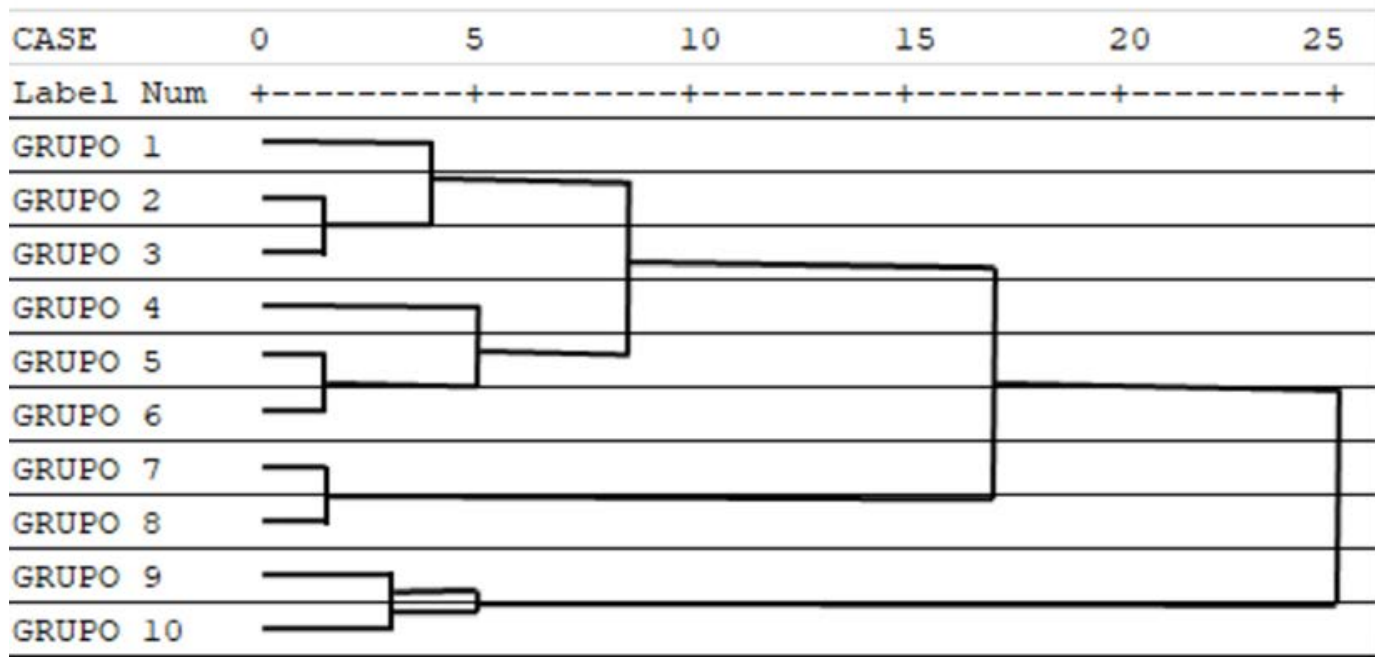

